\title{
Obstetric complications and cognitive function in schizophrenia
}

\author{
Poster Number: CG17P-0914
}

\author{
G. Mezquida (1), E. Fernandez-Egea (2,3,4), D. Treen (5), A. Mané (2,5,6), D. Bergé (5,6,7,8), L. García-Alvarez (2, 9,10) \\ P. García-Portilla $(2,9,10)$, J Bobes $(2,9,10)$, M. Bernardo $(1,2,11,12)$, C. García-Rizo $\left(1,2,11,12,{ }^{*}\right)$
}

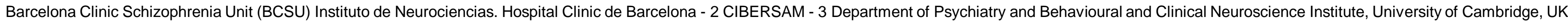

4 Cambridgeshire and Peterborough NHS foundation Trust, United Kingdom - 5 Institut de Neuropsiquiatria i Addiccions, Hospital del Mar, Barcelona - 6 Fundació IMIM, Barcelona - 7 Departament de Psiquiatria i Medicina Legal, Universitat Autónoma de Barcelona - 8 Neuropharmacology, Universitat Pompeu Fabra, Barcelona - 9 Instituto de Neurociencias del Principado de Asturias (INEUROPA), Area de Psiquiatría, Universidad de Oviedo, Asturias -

\section{Background:}

Cognitive function in adulthood is a complex interaction of neuronal pathways and neuroanatomic structures essential in human development. Although diverse factors have been described to interfere in cognitive function, several lines evidence support the fact that early life stressful events (abnormal intrauterine growth, infections, and placenta abnormalities) predict future cognitive impairments in adulthood.

Currently, it is also considered that the perinatal stage can substantially modify the possibility of presenting different pathologies in adulthood. In parallel, the diagnosis of schizophrenia has been associated with a higher prevalence of perinatal stressors: obstetric complications, prenatal infections, labor difficulties, and postpartum complications [1]. Nowadays, the influence of perinatal complications of the patient with schizophrenia has expanded not only to diagnosis per se [2], but also to symptomatology, neuroimaging, metabolism [3] and cognitive function of the patient in adulthood.

Thus, this study aimed to establish a specific cognitive pattern in between the patients with schizophrenia that might be related to early environmental disturbances, as obstetric complications.

\section{Methods:}

In this cross-sectional and international multicenter study, the obstetric complications (OC) suffered by 60 patients diagnosed with schizophrenia (according to DSM-IV) and their correlation with cognitive performance were evaluated.

Obstetric complications were recorded using the Lewis-Murray Scale. Due to epidemiological reasons, the scale was divided into three categories previously described in the literature (factors that occurred during the childbirth, factors that condition low birth weight and stressors during pregnancy). Cognitive function was assessed using the MATRICS Consensus Cognitive Battery (MCCB).

\section{Results:}

The socio-demographic and clinical characteristics of the sample are presented in the Table 1. In our schizophrenia sample, patients who had experienced factors related to low birth weight $(\mathrm{N}=17)$ with respect to patients who had not experienced these factors $(\mathrm{N}=43) \mathrm{had}$ significant differences in cognitive function. Specifically, these differences were found in working memory $(t=-2.34, p=0.02)$ and in verbal learning/recall $(t=-$ 3.29, $p=0.002$ ) (figure
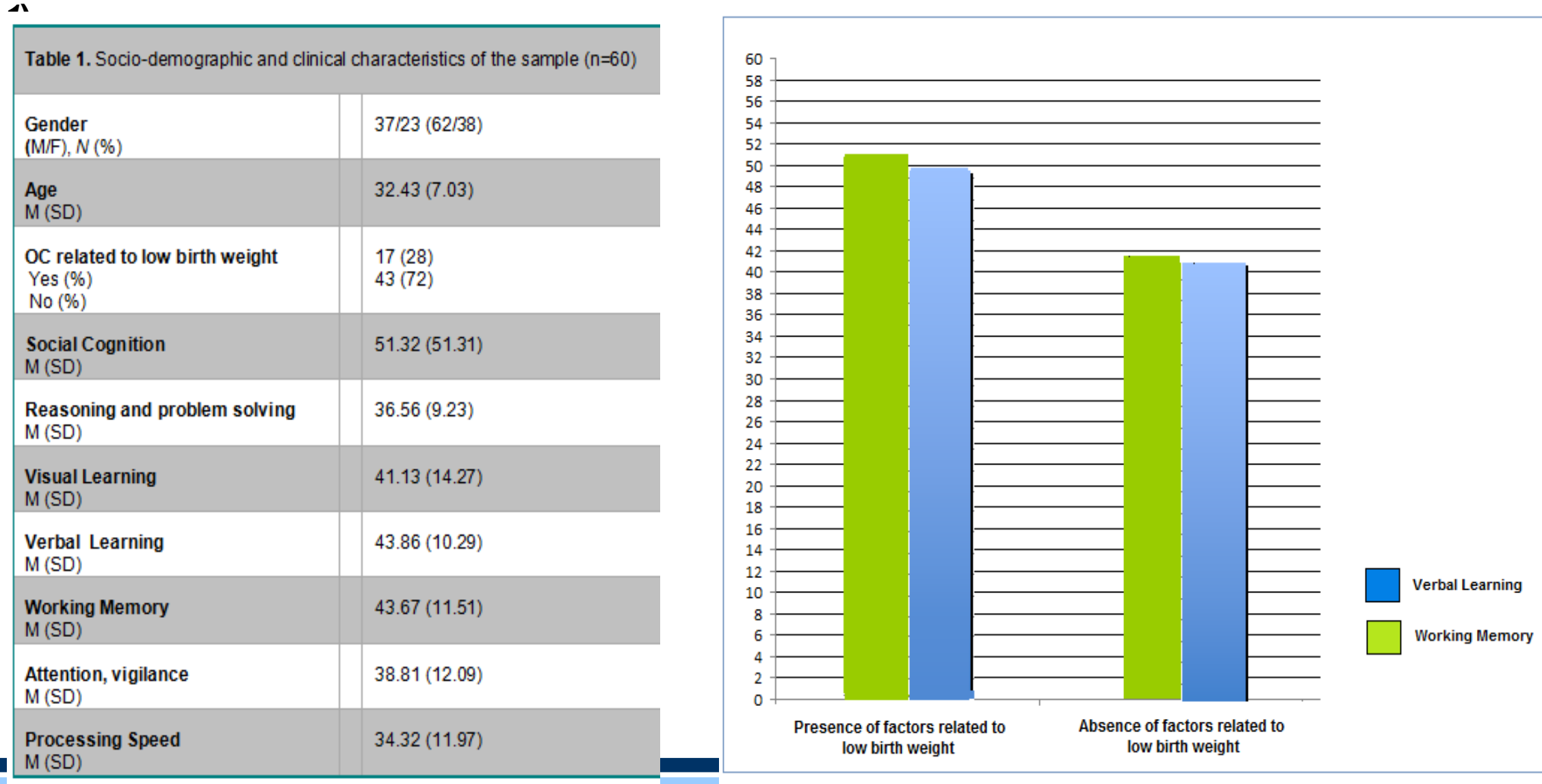

Figure 1. Differences between presenting or not $\mathrm{OC}$ related to low birth weight in Working memory and Verbal learning

\section{Conclusions:}

Cognitive function in schizophrenia, which is considered one of the main predictors of functionality, may be influenced by perinatal factors. To date, only a few previous studies have directly investigated the relationship between these neurodevelopmental risk factors and cognitive deficits in schizophrenia. In our case, contrary to previous literature and our expectations, the presence of complications during pregnancy has shown to be associated with better cognitive functioning in two domains: working memory and verbal learning/recall.

The pathophysiological substrate of our results maintains the same line of gene-environment interaction [4]. Although initially it may seem contradictory, patients who have not suffered environmental factors, we presume a greater genetic cause of the disease and a worse cognitive functioning. Thus, the evaluation of perinatal environmental stressors and the understanding of how these are related to cognition broaden new fields of research in the study of the heterogeneity underlying schizophrenia. These data may also facilitate a better understanding of the etiopathogenesis of the cognitive underpinnings of schizophrenia [5], which is an essential element for the development of primary prevention and individualized intervention strategies.

\section{References:}

